\title{
Clinical and laboratory features of 103 patients from 42 Italian families with inherited thrombocytopenia derived from the monoallelic Ala156Val mutation of GPlb $\alpha$ (Bolzano mutation)
}

Patrizia Noris, ${ }^{1}$ Silverio Perrotta, ${ }^{2}$ Roberta Bottega,${ }^{3}$ Alessandro Pecci, ${ }^{1}$ Federica Melazzini, ${ }^{1}$ Elisa Civaschi, ${ }^{1}$ Sabina Russo, ${ }^{4}$ Silvana Magrin, ${ }^{5}$ Giuseppe Loffredo, ${ }^{6}$ Veronica Di Salvo, ${ }^{7}$ Giovanna Russo, ${ }^{8}$ Maddalena Casale, ${ }^{2}$ Daniela De Rocco, ${ }^{3}$ Claudio Grignani, ${ }^{1}$ Marco Cattaneo, ${ }^{9}$ Carlo Baronci, ${ }^{10}$ Alfredo Dragani, ${ }^{11}$ Veronica Albano, ${ }^{12}$ Momcilo Jankovic, ${ }^{13}$ Saverio Scianguetta, ${ }^{2}$ Anna Savoia, ${ }^{3}$ and Carlo L. Balduini ${ }^{1}$

${ }^{1}$ Istituto di Ricovero e Cura a Carattere Scientifico Policlinico San Matteo Foundation, University of Pavia, Pavia; ${ }^{2}$ Department of Paediatrics, Second University of Napoli, Napoli; 'Institute for Maternal and Child Health - IRCCS "Burlo Garofolo", University of Trieste, Trieste; ${ }^{4}$ Division of Hematology, Department of Internal Medicine, University of Messina, Messina; ${ }^{5}$ Division of Hematology and BMT Unit, V. Cervello Hospital, Palermo; ${ }^{6}$ Department of Oncology, Azienda Santobono-Pausilipon, Pausilipon Hospital, Napoli; ${ }^{7}$ Division of Hematology and Blood Rare Disorders, AOR Villa Sofia-V. Cervello, Palermo; ${ }^{8}$ Division of Pediatric Hematology/Oncology, University of Catania, Catania; 'Unità di Medicina 3, Ospedale San Paolo, Dipartimento di Medicina, Chirurgia e Odontoiatria, Università di Milano, Milano; ${ }^{10}$ Department of Oncohematology, Children's Hospital Bambino Gesù, Roma; ${ }^{11}$ Dipartimento di Ematologia, Servizio di Prevenzione e Cura Sindromi Emorragiche e Trombotiche, Ospedale Civile dello Spirito Santo, Pescara; ${ }^{12}$ Oncoematologia Pediatrica, Istituto di Clinica Pediatrica, Università di Ancona, Ancona, and ${ }^{13}$ Pediatrics Clinic, University of Milano-Bicocca, Hospital San Gerardo, MBBM Foundation, Monza, Italy

ABSTRACT

\section{Background}

Bernard-Soulier syndrome is a very rare form of inherited thrombocytopenia that derives from mutations in GPIb $\alpha, \mathrm{GPIb} \beta$, or GPIX and is typically inherited as a recessive disease. However, some years ago it was shown that the monoallelic c.515C>T transition in the GPIBA gene (Bolzano mutation) was responsible for macrothrombocytopenia in a few Italian patients.

\section{Design and Methods}

Over the past 10 years, we have searched for the Bolzano mutation in all subjects referred to our institutions because of an autosomal, dominant form of thrombocytopenia of unknown origin.

\section{Results}

We identified 42 new Italian families (103 cases) with a thrombocytopenia induced by monoallelic Bolzano mutation. Analyses of the geographic origin of affected pedigrees and haplotypes indicated that this mutation originated in southern Italy. Although the clinical expression was variable, patients with this mutation typically had a mild form of Bernard-Soulier syndrome with mild thrombocytopenia and bleeding tendency. The most indicative laboratory findings were enlarged platelets and reduced GPIb/IX/V platelet expression; in vitro platelet aggregation was normal in nearly all of the cases.

\section{Conclusions}

Our study indicates that monoallelic Bolzano mutation is the most frequent cause of inherited thrombocytopenia in Italy, affecting $20 \%$ of patients recruited at our institutions during the last 10 years. Because many people from southern Italy have emigrated during the last century, this mutation may have spread to other countries.

Key words: inherited thrombocytopenia, Bolzano mutation, monoallelic, Bernard-Soulier syndrome

Citation: Noris P, Perrotta S, Bottega R, Pecci A, Melazzini F, Civaschi E, Russo S, Magrin S, Loffredo G, Di Salvo V, Russo G, Casale M, De Rocco D, Grignani C, Cattaneo M, Baronci C, Dragani A, Albano V, Jankovic $M$, Scianguetta S, Savoia A, and Balduini CL. Clinical and laboratory features of 103 patients from 42 Italian families with inherited thrombocytopenia derived from the monoallelic Ala156Val mutation of GPIba (Bolzano mutation). Haematologica 2012;97(1):82-88. doi:10.3324/haematol.2011.050682

(C)2012 Ferrata Storti Foundation. This is an open-access paper.
Acknowledgments: we thank all patients and their families for participating in the project. We acknowledge Dr. Laura Maria Ciardelli (Department of Microbiology, IRCCS Policlinico San Matteo Foundation, University of Pavia, Pavia, Italy) who performed platelet counts using automated blood cell analyzers in patients enrolled in Pavia, Irene Zorzoli (Istituto di Ricovero e Cura a Carattere Scientifico Policlinico San Matteo Foundation, University of Pavia, Pavia, Italy) for help in the molecular analysis and Dr. Giovanni Amendola (Department of Pediatrics and Neonatology, Umberto I Hospital, Nocera Inferiore, Salerno, Italy) for help in collecting clinical data of patients enrolled at the Second University of Naples.

Funding: this study was supported by grants from the Italian Ministry of Education, University and Research (PRIN 2009), the IRCCS Burlo Garofolo (Grant N 32/09), and Italian ISS (Istituto Superiore di Sanità; Grant: Italian/USA Rare Diseases).

Manuscript received on June 27, 2011. Revised version arrived on August 31, 2011. Manuscript accepted on September 19, 2011.

\section{Correspondence:}

Carlo L. Balduini, Clinica Medica III, Fondazione IRCCS Policlinico San Matteo, Università di Pavia, Piazzale Golgi, 27100 Pavia,

Italy.

E-mail: c.balduini@smatteo.pv.it 


\section{Introduction}

Bernard-Soulier syndrome (BSS) is an inherited, nonsyndromic platelet disorder that is characterized by quantitative and qualitative platelet defects. BSS is caused by mutations in the genes for glycoprotein (GP) Ib $\alpha$, GPI $\beta$ and GPIX, which result in defective GPIb/IX/V complex expression on the platelet surface and/or a reduced ability of GPIb $\alpha$ to interact with von Willebrand factor (vWF). ${ }^{1}$ BSS is considered to be an exceedingly rare disease because it affects less than one subject per million people. ${ }^{2}$ The syndrome has been historically described as an autosomal recessive disease, and more than 50 different mutations have been identified in homozygous or double-heterozygous patients (Bernard-Soulier Syndrome Website and Registry). ${ }^{3}$ However, a few reports have suggested that BSS may occasionally be transmitted in an autosomaldominant fashion. ${ }^{4-6}$ In 2001, we described six Italian families that had been previously diagnosed with Mediterranean macrothrombocytopenia and had the monoallelic c.515C > T transition in the GPIBA gene; ${ }^{7}$ this genetic mutation results in a p.Ala156Val substitution (Bolzano mutation), which reduces the ability of GPIb $\alpha$ to interact with $\mathrm{vWF}^{8}$

Since 2001, we have included the search for monoallelic Bolzano mutation among the diagnostic tests for subjects with non-syndromic, inherited thrombocytopenias. We report here 103 new patients with the monoallelic Bolzano mutation and describe their clinical symptoms and laboratory findings. Moreover, we present data suggesting that this mutation originated in an ancestral individual in southern Italy.

\section{Design and Methods}

\section{Patients}

We enrolled 216 patients in this study who were referred to the IRCCS Policlinico San Matteo Foundation of Pavia or to the Department of Pediatrics of the Second University of Naples between 2001 and 2010 because of a dominant, non-syndromic form of inherited thrombocytopenia of unknown origin.

The institutional review boards of both institutions approved the study, and all patients gave written informed consent in accordance with the Declaration of Helsinki.

\section{Genetic analyses DNA isolation}

Genomic DNA was isolated from blood samples that were anticoagulated with ethylene-diamine-tetra-acetic acid (EDTA) according to standard blood collection procedures.

\section{Mutation screening}

To detect the c.515C $>\mathrm{T}$ transition, polymerase chain reaction (PCR) products were amplified from genomic DNA with the primers $1 \mathrm{~F}$ (5'-CCCTGCGTGGTCTTGGCA-3') and 1R (5'-ATAGAGGATCTCACAGTTGC-3'); the amplicons were subsequently digested with the restriction enzyme HpaI, as previously described?

\section{Haplotype analysis}

The haplotypes were derived by analyzing two polymorphic intragenic markers: the single nucleotide polymorphism (SNP) rs6065 and a variable number of tandem repeats (VNTR) locus. The SNP locus was amplified with the primers $2 \mathrm{~F}$ (5'-
ACACTTCACATGACTCCAT-3') and 1R, and the PCR product was sequenced with an ABI PRISM 3130xl (Applied Biosystems). The VNTR locus was amplified with the primers $2 \mathrm{~F}$ and $2 \mathrm{R}$ (5'GGGTCATTTCTGGA GCTCTC-3'), and the amplicon was analyzed on a $2.5 \%$ agarose gel.

\section{$\beta 1$-tubulin polymorphism}

The $\beta 1$-tubulin gene (TUBB1) region that includes exon 2 and the double base-pair substitution (AG to CC at nucleotide positions 130-131), which encodes for the Q43P mutant form of the protein, was PCR amplified with a primer set described previously. The polymorphism was genotyped by PvuII (Fermentas, Vilnius, Lithuania) restriction digestion and electrophoresis on a $10 \%$ acrylamide gel.

\section{Bleeding tendency}

The bleeding tendency was measured according to the World Health Organization (WHO) bleeding scale (grade 0, no bleeding; grade 1 , petechiae; grade 2 , mild blood loss; grade 3 , gross blood loss; and grade 4, debilitating blood loss).

\section{Platelet count}

EDTA-anticoagulated blood samples were used to determine the platelet count, which was performed within $2 \mathrm{~h}$ of drawing blood. The Sysmex XE-2100 ${ }^{\circledR}$ (Sysmex Corporation, Kobe, Japan) analyzer was used to measure the platelet count based on the impedance method. In some patients the platelet count was also measured with an ADVIA $120^{\circledR}$ counter (Bayer, Tarrytown, NY, USA), which uses two-dimensional laser light-scatter, as well as by manual platelet counting with an optical microscopy in a Neubauer chamber as recommended by the International Committee for Standardization in Haematology. ${ }^{10}$

\section{Platelet size}

The mean platelet volume was measured in parallel with the platelet count with the two analyzers described above. The platelet diameter was measured by optical microscopy on MayGrünwald-Giemsa-stained peripheral blood films, and the pictures were analyzed with Axio-vision $4.5^{\circledR}$ software (Carl Zeiss, Göttingen, Germany). ${ }^{11}$ The blood smears were prepared from non-anticoagulated blood, and the largest diameter of each platelet was measured. The mean platelet diameter was calculated from 200 different cell measurements.

\section{Platelet aggregation}

To analyze in vitro platelet aggregation, blood was collected in $3.8 \%(\mathrm{w} / \mathrm{v})$ sodium citrate (blood:anticoagulant ratio 9:1). To minimize the loss of denser platelets, platelet-rich plasma (PRP) was obtained by sedimentation of the blood at $1 \mathrm{~g}$ for 20 to $30 \mathrm{~min}$. Platelet aggregation was evaluated by the densitometric method according to Born, ${ }^{12}$ after stimulating the PRP with collagen (4 $\mu \mathrm{g} / \mathrm{mL}$ ) (Mascia Brunelli, Milan, Italy), adenosine diphosphate (ADP $5 \mu \mathrm{M}$, Sigma-Aldrich, St. Louis, MO, USA) or ristocetin (1.5 $\mathrm{mg} / \mathrm{mL}$, Sigma-Aldrich). Platelets that did not aggregate in response to ristocetin $1.5 \mathrm{mg} / \mathrm{mL}$ were also tested with a higher dose $(3 \mathrm{mg} / \mathrm{mL})$. The extent of platelet aggregation was measured 5 min after the addition of the stimulating agents, and the results obtained for the patients were compared to the normal range in our laboratory.

\section{Platelet flow cytometry}

The PRP obtained from EDTA-anticoagulated blood was used for flow cytometric analysis of surface platelet glycoprotein expression as previously reported. ${ }^{7}$ The platelets were incubated with the following monoclonal antibodies: SZ2 (Immunotech, 
Marseille, France) and MB45 (CLB, Amsterdam, the Netherlands), which bind GPIba (CD42b); SZ1 (Immunotech), which binds GPIX (CD42a); SW16 (CLB), which binds GPV (CD42d); SZ21 (Immunotech), which binds GPIIIa (B3/CD61); P2 (Immunotech), which binds GPIIb ( $\alpha \mathrm{IIb})$ when it is correctly complexed with GPIIIa; and Gi9 (Immunotech), which binds GPIa (CD49b). $\mathrm{MO}_{2}$ was used as a negative control. $\mathrm{MO}_{2}$ and a fluorescein isothiocyanate-conjugated goat anti-mouse $\operatorname{IgG}$ were purchased from Coulter (Coulter Corporation, Miami, USA). Fluorescent antibody staining of the platelets was analyzed with an Epics XL flow cytometer (Coulter).

\section{Serum thrombopoietin levels}

The serum thrombopoietin level was determined with a commercially available ELISA kit (Quantikine Human TPO Immunoassay, R\&D Systems, Minneapolis, USA) according to the manufacturer's instructions. The detection limits of this assay are between 7 and 2,000 pg/mL of serum. For the purposes of this study, a value of $6.9 \mathrm{pg} / \mathrm{mL}$ was assigned to samples in which the thrombopoietin concentration was lower than the detection limit.

\section{Statistical analysis}

Continuous data are presented as means, standard deviations (SD), and ranges. Categorical variables are reported as counts and percentages. Laboratory parameters were compared with the mean value of the reference population with a parametric test for paired values (Student's $t$ test).

\section{Results}

\section{Genetic studies}

\section{Mutation screening}

Screening for the c.515C>T mutation in 216 affected individuals with a non-syndromic, autosomal-dominant form of macrothrombocytopenia identified 42 carriers. Genetic molecular testing in all of the available relatives indicated that this mutation was present in 61 additional individuals, all of whom had low platelet counts as measured by the impedance cell counter. The platelet count was normal in all of the relatives who did not have the Bolzano mutation.

\section{Haplotype analysis}

To determine whether the mutation occurred as a unique mutational event, we genotyped two polymorphic loci flanking the c.515C>T mutation: the SNP rs6065 $(\mathrm{C}>\mathrm{T})$, which is responsible for a threonine to methionine mutation at amino acid 145 of the human platelet antigen 2 (HPA2) protein, and a size polymorphism in the GP1BA coding region that contains a VNTR ranging in size from one to four repeats of 39 nucleotides (alleles A, 4 tandem repeats, to $D$, one tandem repeat). ${ }^{13}$

In five families in which both of the parents of the patient were analyzed, all of the affected individuals shared the same haplotype (T-T-B), which suggests a common ancestral chromosome. Moreover, genotyping of 74 patients from the other pedigrees showed that two of them were homozygous for the T allele of SNP rs6065 and the $\mathrm{B}$ variant of the VNTR; the remaining patients were all carriers of at least one T allele of SNP rs6065 and B variant of the VNTR. Since the frequency of the $T$ allele of SNP rs6065 and the B variant of VNTR in European populations is $10 \%$ and $12 \%$, respectively, while the frequency of the C variant of SNP rs6065 and the A variant of VNTR is $81 \%$ and $7 \%$, respectively (HapMap), all patients are likely to share the same mutated haplotype.

\section{$\beta 1$-tubulin polymorphism}

$\beta 1$-tubulin plays a role in proplatelet formation, ${ }^{14}$ as well as in maintaining the discoid platelet shape and platelet function. The mutant $\beta 1$-tubulin protein Gln43Pro has been associated with abnormal platelet morphology, in vitro defects of platelet function, ${ }^{9}$ and an increased risk of intracerebral hemorrhage. ${ }^{15}$ We hypothesized that this mutation may cause the variation in platelet count, platelet size and bleeding tendency observed in subjects with the Bolzano mutation (see below). We, therefore, genotyped all patients carrying the Bolzano mutation and identified 22 homozygotes and 81 heterozygotes for the Gln43Pro mutation. However, there was no significant difference in the mean platelet diameter, the degree of thrombocytopenia or the bleeding score between these two genotypes (data not shown).

\section{Clinical picture}

Fifty-three patients with the Bolzano mutation were female and 50 were male. Thirty-two families were native to southern Italy, mostly from Campania and Sicily, while the remaining 10 families were apparently from northern Italy. The patients' mean age was 33 years (range, 1-93 years). Prior to identification of the genetic etiology of thrombocytopenia, 13 patients were diagnosed as suffering from immune thrombocytopenia and five of them were treated with steroids, intravenous immunoglobulins and/or splenectomy.

The patients' bleeding tendency was measured according to the WHO bleeding scale and is reported in Table 1. The bleeding scores were quite variable, with $41.7 \%$ of the cases presenting recurrent, spontaneous hemorrhage. None of the patients experienced abnormal bleeding associated with dental extractions or minor/major surgery. Epistaxis, gum bleeding, easy bruising and menorrhagia were the most common symptoms. Severe hemorrhagic episodes included rectal bleeding that required a platelet transfusion (one patient), post-traumatic brain hemorrhage (one patient), epistaxis requiring medical intervention (two patients), and post-partum hemorrhage requiring surgical intervention (one patient). Sixty patients $(58.2 \%)$ had no history of bleeding. Overall, 19 women delivered 32 children (vaginal and Cesarean section births) with no platelet transfusions, and no excessive bleeding was reported; another patient received prophylactic platelet concentrates prior to two deliveries, although she had never experienced a bleeding episode.

No correlation between bleeding tendency and any of the investigated laboratory features was identified.

\section{Platelet count}

According to the data from the impedance counter, all of the subjects with the c.515C>T mutation had platelet counts lower than $150 \times 10^{9} / \mathrm{L}$; however, thrombocytopenia was usually mild (mean platelet count $81 \times 10^{9} / \mathrm{L}$ ) and only 18 patients had a platelet count lower than $50 \times 10^{9} / \mathrm{L}$. Moreover, a comparison of the platelet count obtained by three independent methods in 23 patients (Table 2) revealed that the impedance counter overestimated the degree of thrombocytopenia with respect to both the optical counter and manual counting in a Neubauer chamber $(P<0.001$ for both methods). Of note, optical counting and 
manual counting revealed that, respectively, two and three of the 23 subjects who were classified as thrombocytopenic based on impedance counting actually had platelet counts at the lower limit of the normal range. This discrepancy is not surprising because it is well known that electronic counters often fail to recognize and count large platelets; this problem is more common with impedance instruments than with optical counters. Thus, these laboratory instruments often underestimate the platelet count in subjects with macrothrombocytopenia. ${ }^{11}$

\section{Platelet size}

Quantification of the mean platelet diameter in 81 patients (Table 1) revealed that the patients' platelets were $50 \%$ larger than the platelets from controls $(P<0.001)$. This difference was even greater when the mean platelet volume was analyzed (Table 2): the mean platelet volume in the 23 patients who had their platelets evaluated by the optical counter was $83 \%$ higher than that of the controls $(P<0.001)$. The impedance counter did not calculate a mean platelet volume for the majority of the patients because there were abnormalities in their platelet volume distribution curves (data not shown); thus, impedance counters are limited in their ability to evaluate macrothrombocytopenias.

\section{In vitro platelet aggregation}

In vitro platelet aggregation in response to $\mathrm{ADP}$ and collagen was within the normal range for all of the 23 patients studied. Platelet aggregation in response to ristocetin $1.5 \mathrm{mg} / \mathrm{mL}$ (Table 2) was decreased in five patients (mean increase in light transmittance $22 \pm 11.4 \%$ ) and nor$\mathrm{mal}$ in the remaining 18 subjects (mean increase in light transmittance $82 \pm 13.6 \%$; range for normal subjects $50-$ $100 \%)$. All of the five patients who did not respond normally to ristocetin $1.5 \mathrm{mg} / \mathrm{mL}$ showed a normal response after stimulation with $3 \mathrm{mg} / \mathrm{mL}$ ristocetin. The normal aggregation in response to the high dose of ristocetin and the observation that the GPIb/IX/V complex was about $50 \%$ of control (see below) excluded that these five subjects were affected by a biallelic form of BSS.

\section{Platelet flow cytometry}

Flow cytometry analysis of platelet receptor expression levels was performed in 37 patients from 16 families. Monoclonal antibody binding (Figure 1) indicated that GPIIb-IIIa and GPIa expression was slightly increased compared to that of the controls $(P<0.05$ and $P<0.001$, respectively), while the level of expression of all the GPIb/IX/V complex components was significantly reduced $(P<0.001)$. These results corroborate our previous data from four Bolzano patients ${ }^{7}$ with the only notable exception that the binding of SZ2 and MB45 to GPIb $\alpha$ was reduced to a similar extent while in our previous study the binding of SZ2 was lower than that of MB45, which led us to hypothesize that the MB45 was less sensitive to the conformational change in the protein caused by the Bolzano mutation.

\section{Serum thrombopoietin level}

The serum thrombopoietin level was measured in 46 patients. The mean value for the patients $(24.6 \pm 21.9$ $\mathrm{pg} / \mathrm{mL}$, range $6.9-64.5 \mathrm{pg} / \mathrm{mL}$ ) was approximately twofold greater than that of 50 healthy subjects $(14 \pm 10.8$ $\mathrm{pg} / \mathrm{mL}$, range 6.9-54.4 $\mathrm{pg} / \mathrm{mL})$. Interestingly, the throm-
Table 1. Clinical and laboratory findings of 103 Bolzano patients from 42 Italian families.

\begin{tabular}{|c|c|c|c|c|}
\hline $\begin{array}{l}\text { Family } \\
\text { (N. of } \\
\text { patients) }\end{array}$ & $\begin{array}{l}\text { Age/year } \\
\text { Mean } \\
\text { (range) }\end{array}$ & $\begin{array}{l}\text { WHO bleeding } \\
\text { score } \\
\text { (N. of patients) }\end{array}$ & $\begin{array}{l}\text { Platelet count by } \\
\text { impedance counter, } \\
\text { x10 } / \text { L Mean } \\
\text { (range) }\end{array}$ & $\begin{array}{l}\text { Mean platelet } \\
\text { diameter, } \mu \mathrm{m} \\
\text { Mean (range) }\end{array}$ \\
\hline $1(2)$ & $28(15-41)$ & 0,2 & $97(69-125)$ & $3.6(3.4-3.9)$ \\
\hline $2(2)$ & $39(18-60)$ & $2(2)$ & 78 (61-95) & $3.6(2.5-4.6)$ \\
\hline $3(2)$ & $25(15-35)$ & 0,2 & $86(85-87)$ & $3.2(3.1-3.4)$ \\
\hline $4(3)$ & $40(29-60)$ & $2(2), 3$ & 75 (47-91) & $3.7(3.1-4.2)$ \\
\hline $5(2)$ & 27 (11-44) & $1(2)$ & 100 (93-108) & $3.1(3.1-3.2)$ \\
\hline $6(1)$ & 26 & 3 & 79 & 3.7 \\
\hline 7 (2) & $16(1-31)$ & $0(2)$ & $85(66-104)$ & $2.9(2.3-3.4)$ \\
\hline $8(1)$ & 53 & 0 & 91 & 3.2 \\
\hline $9(2)$ & $16(1-32)$ & $0(2)$ & $103(100-106)$ & $3.6(3.3-3.9)$ \\
\hline $10(2)$ & $21(3-39)$ & 0,1 & 38 (27-49) & $3.5(3.3-3.7)$ \\
\hline $11(3)$ & $38(15-60)$ & $0(3)$ & $85(44-110)$ & $3.8(3.5-4.3)$ \\
\hline $12(3)$ & $36(25-55)$ & $0(3)$ & $42(30-58)$ & nd \\
\hline $13(1)$ & 7 & 1 & 87 & 3.3 \\
\hline $14(10)$ & $50(8-93)$ & $0(5), 1(5)$ & 65 (30-97) & $3.1(2.9-3.3)^{\dagger}$ \\
\hline
\end{tabular}

\begin{tabular}{ccccc}
\hline $15(1)$ & 51 & 0 & 61 & 4 \\
$16(1)$ & 34 & 0 & 92 & 3.3 \\
\hline $17(2)$ & $23(12-35)$ & 0,2 & $100(93-107)$ & $3.3(3.2-3.3)$ \\
$18(8)$ & $35(1-65)$ & $0,2(5), 3,4$ & $85(21-130)$ & $3.7(3.2-4.1)$ \\
\hline $19(9)$ & $32(10-59)$ & $0(5), 1(4)$ & $91(6-127)$ & nd \\
$20(1)$ & 35 & 1 & 138 & 3 \\
\hline $21(3)$ & $12(2-30)$ & $0,1,2$ & $93(54-115)$ & $3(2.9-3.1)$ \\
$22(2)$ & $19(8-31)$ & $0(2)$ & $84(78-90)$ & $3.4(3.1-3.6)$ \\
\hline $23(2)$ & $33(32-35)$ & $0(2)$ & $113(103-124)$ & $3.5(3.5-3.6)$ \\
$24(3)$ & $34(22-51)$ & $0(2), 2$ & $64(54-73)$ & $3.7(3.2-4.4)$
\end{tabular}

\begin{tabular}{lllll}
\hline $25(1)$ & 21 & 0 & 79 & 3.1
\end{tabular}

$\begin{array}{lllll}26(1) & 24 & 0 & 110 & 3.2\end{array}$

$\begin{array}{lllll}27(1) & 51 & 0 & 98 & 3.9\end{array}$

$\begin{array}{lllll}28(1) & 76 & 4 & 45 & 4.7\end{array}$

$29(2) \quad 44(31-57) \quad 0(2) \quad 29(16-42) \quad 3.9(3.6-4)$

$30(2) \quad 41(30-53) \quad 0,2 \quad 54(50-58) \quad 4.5(4-5.1)$

\begin{tabular}{ccccc}
\hline $31(1)$ & 28 & 3 & 106 & 3.4 \\
$32(3)$ & $35(3-66)$ & $0,1,3$ & $82(34-130)$ & $3.2(3.2-3.4)$
\end{tabular}

$33(2) \quad 17(3-32) \quad 0(2) \quad 111(93-129) \quad 3.7^{\dagger \dagger}$

$34(2) \quad 21(4-39) \quad 0,1 \quad 59(49-69) \quad 3.6^{\text {ti }}$

$35(2) \quad 13(2-24) \quad 0(2) \quad 95(88-103) \quad 3(2.7-3.5)$

$36(3) \quad 37(4-70) \quad 0,2,3 \quad 134(112-147) \quad 3(2.7-3.3)$

\begin{tabular}{llllr}
\hline $37(1)$ & 34 & 0 & 52 & 3.5
\end{tabular}

$38(3) \quad 25(14-45) \quad 0,1,2 \quad 110(101-124) \quad 2.9(2.5-3.4)$

$39(4) \quad 28(8-60) \quad 0(4) \quad 90(57-138) \quad 3.2(2.7-3.7)$

$40(2) \quad 37(25-50) \quad 0(2) \quad 45(37-53) \quad 3.8(3.3-4.2)$

$41(2) \quad 33(20-46) \quad 0(2) \quad 85(76-94) \quad 3.6(3.5-3.7)$

$\begin{array}{lllll}42(2) & 39(23-55) & 0,2 & 56(28-84) & 3.7(3.6-3.8)\end{array}$

All patients $33 \pm 20(1-93) \quad 0(60), 1(18), \quad 82 \pm 32(6-147)^{*} \quad 3.5 \pm 0.5(2.3-5.1)^{*}$ Mean \pm SD $2(17), 3(6)$, (range) $\quad 4(2)$

Controls $32 \pm 14(19-79) \quad 0 \quad 255 \pm 51(141-396) \quad 2.4 \pm 0.3(1.9-3.4)$ Mean \pm SD (range)

${ }^{*} P<0.001$ compared to normal subjects, ${ }^{\prime}$ data obtained in two subjects, ${ }^{\prime \prime}$ data obtained in one subject; nd: not done. 
bopoietin level that we measured in our Bolzano patients is similar to that recently observed in a case series of patients with idiopathic thrombocytopenic purpura $(38.3 \pm 46.0 \mathrm{pg} / \mathrm{mL}){ }^{16}$ Because thrombopoietin is internalized and degraded by platelets and megakaryocytes, ${ }^{17}$ the thrombopoietin serum concentration is inversely related to the total megakaryocyte and platelet mass. Therefore, our data indicate that Bolzano patients do not have a reduced megakaryocyte mass.

\section{Discussion}

The history of inherited thrombocytopenias is strictly connected to that of platelet counting. Until the introduction of automated platelet counters in the late 1970s, the platelet count was visually quantified with a microscope and a counting chamber. Because this method is time-consuming, platelet counts were usually performed only on patients with a bleeding diathesis. Thus, only severe forms of inherited thrombocytopenia were diagnosed. The 1990 edition of Williams Textbook of Hematology ${ }^{18}$ describes four inherited thrombocytopenias: BSS, Wiskott-Aldrich syndrome, gray platelet syndrome and
May-Hegglin anomaly. At that time, BSS was described as an autosomal recessive disorder. The widespread use of electronic counters in the 1990s made platelet counting a routine analysis, and several new forms of mild thrombocytopenia with trivial or no bleeding diathesis have since been discovered; currently there are more than 20 forms of inherited thrombocytopenias. In particular, thrombocytopenic patients with monoallelic mutations in GPIbo have been identified, including six Italian families that carry the p.Ala156Val substitution in GPIba. ${ }^{4 \cdot 7}$ BSS is therefore now classified as a recessive disease that can occasionally be inherited as a dominant disease.

Genetic analyses of the Bolzano mutation in our familial case series indicated that this monoallelic mutation was a frequent cause of inherited thrombocytopenia. In fact, our database of 216 cases of inherited thrombocytopenia observed during the last 10 years includes 42 pedigrees with monoallelic, dominant BSS caused by the Bolzano mutation; 22 of the pedigrees have MYH9-related disease, 21 have thrombocytopenia 2 (THC2), ${ }^{16}$ and 10 pedigrees have the classic biallelic, recessive form of BSS. ${ }^{1}$ Thus, in our experience the monoallelic Bolzano mutation is the most frequent cause of inherited thrombocytopenia. Since most of the patients included in our database are of Italian

Table 2. Platelet counts obtained by three different methods, platelet size (MPV and MPD) and platelet aggregation in response to ristocetin (RIPA) $1.5 \mathrm{mg} / \mathrm{mL}$ in 23 patients.

\begin{tabular}{|c|c|c|c|c|c|c|}
\hline $\begin{array}{l}\text { Family/ } \\
\text { subject }\end{array}$ & $\begin{array}{l}\text { Manual platelet } \\
\text { count, } \times 10^{\circ} / \mathrm{L}\end{array}$ & $\begin{array}{l}\text { Impedance platelet } \\
\text { count, } \times 10^{\circ} / \mathrm{L}\end{array}$ & $\begin{array}{l}\text { Optical platelet } \\
\text { count, } \times 10^{9} / \mathrm{L}\end{array}$ & $\begin{array}{c}\text { MPV, fL, } \\
\text { by optical counter }\end{array}$ & $\begin{array}{c}\text { MPD, um, } \\
\text { by optical microscopy }\end{array}$ & $\begin{array}{l}\text { RIPA by } \\
1.5 \mathrm{mg} / \mathrm{mL}\end{array}$ \\
\hline $1 / 1$ & 184 & 125 & 161 & 16 & 3.41 & reduced \\
\hline $1 / 2$ & 98 & 69 & 100 & 16.5 & 3.98 & reduced \\
\hline $4 / 1$ & 134 & 91 & 119 & 14.3 & 4.08 & normal \\
\hline $4 / 2$ & 124 & 87 & 116 & 14.4 & 3.06 & normal \\
\hline $4 / 3$ & 104 & 47 & 93 & 16.6 & 4.2 & normal \\
\hline $6 / 1$ & 92 & 79 & 84 & 14.8 & 3.72 & reduced \\
\hline $15 / 1$ & 68 & 61 & 63 & 17.8 & 4.02 & normal \\
\hline $18 / 1$ & 128 & 130 & 132 & 14.6 & 3.74 & normal \\
\hline $18 / 2$ & 42 & 21 & 21 & 9.7 & 4.11 & normal \\
\hline $18 / 3$ & 164 & 107 & 106 & 13.4 & 3.34 & normal \\
\hline $18 / 4$ & 141 & 101 & 150 & 15.5 & 4.06 & normal \\
\hline $18 / 5$ & 88 & 65 & 90 & 16.7 & 3.87 & normal \\
\hline $18 / 6$ & 90 & 55 & 84 & 15.2 & 3.63 & normal \\
\hline $18 / 7$ & 142 & 102 & 110 & 13.6 & 3.28 & normal \\
\hline $18 / 8$ & 122 & 103 & 106 & 14.8 & 3.6 & normal \\
\hline $30 / 1$ & 64 & 50 & 59 & 16.7 & 5.08 & normal \\
\hline $30 / 2$ & 72 & 58 & 76 & 17.2 & 4.04 & normal \\
\hline $32 / 2$ & 104 & 81 & 92 & 14.7 & 3.39 & reduced \\
\hline $32 / 3$ & 162 & 130 & 135 & 15.2 & 3.2 & normal \\
\hline $33 / 1$ & 88 & 93 & 100 & 14.2 & 3.71 & normal \\
\hline $36 / 1$ & 128 & 112 & 111 & 15.5 & 3.28 & reduced \\
\hline $36 / 2$ & 144 & 147 & 133 & 12.5 & 2.65 & normal \\
\hline $36 / 3$ & 148 & 142 & 144 & 16.7 & 3.19 & normal \\
\hline $\begin{array}{l}\text { All patients } \\
\text { Mean } \pm \text { SD (range) }\end{array}$ & $\begin{array}{l}114 \pm 3.6^{\dagger} \\
(42-184)\end{array}$ & $\begin{array}{c}89 \pm 33.1^{* * \dagger} \\
(21-147)\end{array}$ & $\begin{array}{c}103 \pm 32.1^{* \dagger} \\
(21-162)\end{array}$ & $\begin{array}{c}15 \pm 1.7^{\dagger} \\
(9.7-17.8)\end{array}$ & $\begin{array}{l}3.6 \pm 0.5^{\dagger} \\
(2.6-5.1)\end{array}$ & $\begin{array}{l}\text { normal: } 18 \text { patients } \\
\text { reduced: } 5 \text { patients }\end{array}$ \\
\hline $\begin{array}{l}\text { Controls } \\
\text { Mean } \pm \text { SD (range) }\end{array}$ & $\begin{array}{l}276 \pm 50.1 \\
(170-420)\end{array}$ & $\begin{array}{l}255 \pm 53.2 \\
(141-396)\end{array}$ & $\begin{array}{l}280 \pm 54.3 \\
(164-412)\end{array}$ & $\begin{array}{c}8.2 \pm 0.8 \\
(7.1-11.1)\end{array}$ & $\begin{array}{l}2.4 \pm 0.3 \\
(1.9-3.4)\end{array}$ & normal \\
\hline
\end{tabular}

${ }^{*} \mathrm{P}<0.05$ compared to manual method. ${ }^{*} \mathrm{P}<0.001$ compared to manual method. ${ }^{\mathrm{P}}<0.001$ compared to normal subjects. MPV: mean platelet volume; MPD: mean platelet diameter. 
origin, we are tempted to conclude that BSS due to monoallelic Bolzano mutation is the most frequent inherited thrombocytopenia in Italy. However, we cannot exclude recruitment bias, since we first described this form of thrombocytopenia ${ }^{7}$ and it is, therefore, possible that for this reason patients with this suspected diagnosis have been referred to us more frequently than those with other suspected diagnoses.

Because the carriers of the Bolzano substitution share the rare T-T-B haplotype, it is likely that this mutation arose from a unique event that occurred on an ancestral Italian chromosome. The observation that most of the affected families were from southern Italy further supports this hypothesis of a founder effect. However, the Italian population has emigrated all over the word during the past century. Although no cases of BSS due to the monoallelic Bolzano mutation have been described outside Italy, we cannot exclude the existence of similar undiagnosed and unreported cases in other countries.

The observation that many of our patients were initially misdiagnosed with immune thrombocytopenia indicates that diagnosis of this form of BSS may be difficult. In particular, the genetic origin of thrombocytopenia may not be acknowledged in patients whose low platelet count is incidentally detected during adulthood, especially when they do not have a documented history of thrombocytopenia. Thus, we suggest that the thrombocytopenia caused by the p.Ala156Val mutation should be considered in all cases in which thrombocytopenia is present from birth. Although the presence of large platelets may be indicative of the Bolzano mutation, a platelet size in the upper part of the normal range does not exclude this genetic mutation. Moreover, although some patients with the Bolzano mutation demonstrate reduced aggregation in response to intermediate doses of ristocetin, most patients display a

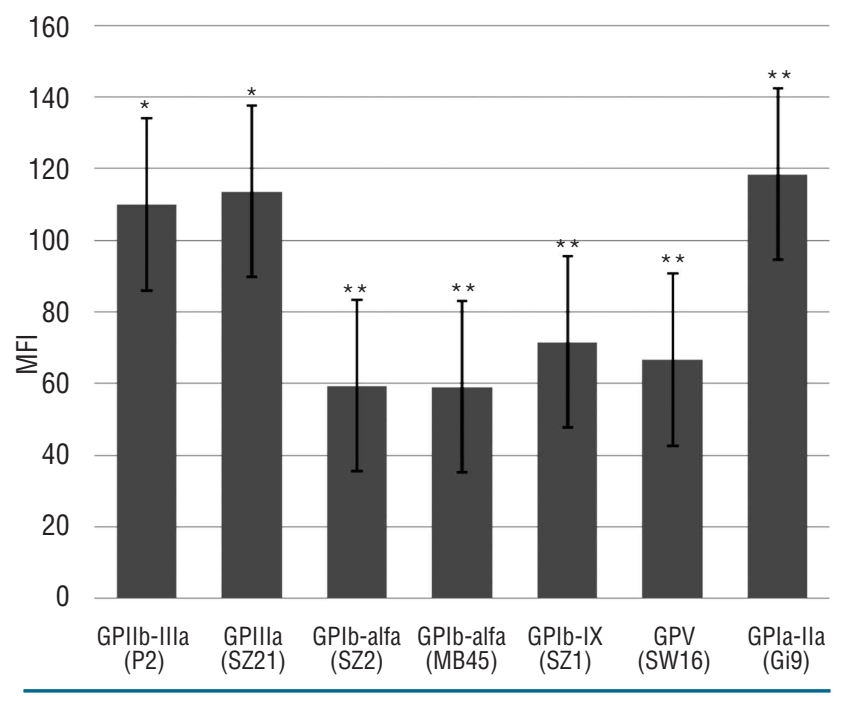

Figure 1. Expression of glycoproteins on the surface platelet membrane in Bolzano patients, measured by flow cytometry. The mean and the standard deviation of the expression of each platelet glycoprotein obtained in 39 Bolzano patients are shown. For each patient the expression of membrane platelet glycoprotein was calculated as the percentage of the mean fluorescent intensity (MFI) obtained for the same monoclonal antibody in the relative control. ${ }^{*} P<0.05$ compared to normal subjects; ${ }^{*} P<0.01$ compared to normal subjects. normal response. Flow cytometry provided the most evocative results because commercially available monoclonal antibodies against the GPIb/IX/V complex components exhibited reduced binding to the patients' platelets. Genotyping is also a useful diagnostic strategy, because the p.Ala156Val mutation can be detected by a restriction enzyme assay, which is a rapid and easy diagnostic assay. Raising the awareness about this form of hereditary thrombocytopenia will certainly increase the rate of correct diagnosis reducing the risk of inappropriate treatments for these patients.

Despite the genetic homogeneity among our patients, the degree of thrombocytopenia, platelet macrocytosis and bleeding tendency were all variable. It has been suggested that the common Gln43Pro variant of $\beta 1$-tubulin is associated with reduced platelet production, abnormal platelet shape and a predisposition to bleeding., ${ }^{9,14}, 15 \mathrm{We}$, therefore, hypothesized that this polymorphism may modulate the BSS phenotype in our patients. However, further genetic analyses for Gln43Pro did not identify a genotype-phenotype correlation. Thus, other as yet unknown genetic and/or environmental factors may modify the phenotype of patients with the monoallelic Bolzano mutation.

One question raised by our study is whether the term BSS is appropriate for patients with the monoallelic Bolzano mutation. BSS is defined as thrombocytopenia with large platelets and platelet dysfunction due to quantitative or qualitative defects in GPIb $\alpha$. The platelet count was low in all but two of the patients with the Bolzano mutation, and their mean platelet diameter was larger than that of controls. Flow cytometry analysis of platelet receptor expression revealed that GPIb $\alpha$ was defective, and the mean extent of platelet aggregation with ristocetin was at the lower limit of the normal range, with $17 \%$ of the investigated subjects demonstrated a clearly decreased response. Moreover, the bleeding tendency in symptomatic patients was disproportionate to the degree of thrombocytopenia, which suggests that platelet dysfunction contributes to the bleeding tendency. Thus, all of the defects that characterize BSS were present in patients with the monoallelic Bolzano mutation, although the symptoms were less severe than those of patients with the recessive biallelic form of the disease. Thus, we suggest that the form of thrombocytopenia described here should be classified as a mild form of BSS. Because the clinical and laboratory features of the few reported cases of thrombocytopenia with monoallelic mutations in GPIba other than the Bolzano mutation are similar to those of our patients, ${ }^{4 \cdot 6}$ we propose that the term monoallelic dominant BSS be used for this mild form of inherited thrombocytopenia. This term would distinguish this form of the disease from that in both the BSS carriers with monoallelic mutations who are asymptomatic and that in patients with the more severe, recessive biallelic form.

Another interesting question is why some monoallelic mutations result in the mild form of BSS while other mutations do not affect the phenotype. Interestingly, microdeletions within chromosome 22q11 which result in the loss of one GPIBB allele (velocardiofacial syndrome) reduce the surface expression of GPIb/IX/V by $50 \%$; however, in most cases, these mutations do not induce thrombocytopenia or platelet macrocytosis. ${ }^{19}$ Thus, $50 \%$ expression of the GPIb/IX/V complex appears to be sufficient for 
normal megakaryopoiesis and platelet production. Based on this observation, it seems reasonable to hypothesize that a few BSS mutations in GPIba, namely the Bolzano mutation, p.Asn41His, ${ }^{6}$ p.Tyr54Asp ${ }^{5}$ and p.Leu57Phe ${ }^{4}$ exert a dominant effect that affects the platelet count and platelet size also when $50 \%$ of the wild-type protein is expressed. In contrast, most BSS mutations do not consistently cause thrombocytopenia in the presence of one normal allele, although some mutations, such as p.Tyr88Cys in GPIb $\beta{ }^{20}$ result in the production of larger platelets. Not enough data are presently available to unravel the molecular mechanisms that underlie these differences, and further studies on the transcription and translation of mutated genes, as well as on the processes that regulate the assembly of the GPIb/IX/V complex, are required to fully understand the pathogenesis of BSS.

In conclusion, our study indicates that the monoallelic
Bolzano mutation is a frequent cause of macrothrombocytopenia in Italy and that this mutation induces a mild form of BSS.

Further analyses in other countries will reveal whether this form of the disease is exclusive to Italy or whether it also affects other populations.

\section{Authorship and Disclosures}

The information provided by the authors about contributions from persons listed as authors and in acknowledgments is available with the full text of this paper at www. haematologica.org.

Financial and other disclosures provided by the authors using the ICMJE (www.icmje.org) Uniform Format for Disclosure of Competing Interests are also available at www. haematologica.org.

\section{References}

1. Savoia A, Pastore A, De Rocco D, Civaschi E, Di Stazio M, Bottega $R$ et al. Clinical and genetic aspects of Bernard-Soulier syndrome: searching for genotype/phenotype correlations. Haematologica. 2011;96(3): 417-23.

2. Lopez JA, Andrews RK, Afshar-Kharghan $\mathrm{V}$, Berndt MC. Bernard-Soulier syndrome. Blood. 1998;91(12):4397-418.

3. Bernard-Soulier Syndrome Website and Registry. http://www.bernardsoulier.org/

4. Miller JL, Lyle VA, Cunningham D. Mutation of leucine-57 to phenylalanine in a platelet glycoprotein Ib $\alpha$ leucine tandem repeat occurring in patients with an autosomal dominant variant of Bernard-Soulier disease. Blood. 1992;79 (2):439-46.

5. Kunishima S, Imai T, Hamaguchi M, Saito $\mathrm{H}$. Novel heterozygous missense mutation in the second leucine rich repeat of GPIbalpha affects GPIb/IX/V expression and results in macrothrombocytopenia in a patient initially misdiagnosed with idiopathic thrombocytopenic purpura. Eur J Haematol. 2006;76(4):348-55.

6. Vettore S, Scandellari R, Moro S, Lombardi AM, Scapin M, Randi ML, et al. Novel point mutation in a leucine-rich repeat of the GPIbalpha chain of the platelet von Willebrand factor receptor, GPIb/IX/V, resulting in an inherited dominant form of Bernard-Soulier syndrome affecting two unrelated families: the $\mathrm{N} 41 \mathrm{H}$ variant. Haematologica. 2008;93(11):1743-7.

7. Savoia A, Balduini CL, Savino M, Noris P,
Del Vecchio M, Perrotta S, et al. Autosomal dominant macrothrombocytopenia in Italy is most frequently a type of heterozygous Bernard-Soulier syndrome. Blood. 2001;97 (5):1330-5.

8. Ware J, Russell SR, Marchese P, Ruggeri ZM. Point mutation in a leucine-rich repeat of platelet glycoprotein Ib alpha resulting in the Bernard-Soulier syndrome. J Clin Invest. 1993;92(3):1213-20.

9. Freson K, De Vos R, Wittevrongel C, Thys C, Defoor J, Vanhees L, et al. The TUBB1 Q43P functional polymorphism reduces the risk of cardiovascular disease in men by modulating platelet function and structure. Blood. 2005;106(7):2356-62.

10. England JM, Rowan RM, Bins M, Bull BS, Coulter WH, Groner W, et al. Recommended methods for the visual determination of white cell and platelet counts. Available at: http://whqlibdoc.who.int/hq/1988/WHO_L AB_88.3.pdf

11. Noris P, Klersy C, Zecca M, Arcaini L, Pecci A, Melazzini F, et al. Platelet size distinguishes between inherited macrothrombocytopenias and immune thrombocytopenia. J Thromb Haemost. 2009;7(12):2131-6.

12. Born GV. Aggregation of blood platelets by adenosine diphosphate and its reversal. Nature. 1962;194:927-9.

13. Di Paola J, Jugessur A, Goldman T, Reiland J, Tallman D, Sayago C, et al. Platelet glycoprotein I(b)alpha and integrin alpha2 beta1 polymorphisms: gene frequencies and linkage disequilibrium in a population diversity panel. J Thromb Haemost. 2005;3(7):151121.
14. Schwer $\mathrm{HD}$, Lecine $\mathrm{P}$, Tiwari S, Italiano JE, Hartwing JH, Shivdasani RA. A lineagerestricted and divergent beta-tubulin isoform is essential for the biogenesis, structure, and function of blood platelets. Curr Biol. 2001;11(8):579-86.

15. Navarro-Nunez L, Lozano ML et al The association of the beta1.tubulin polymorphism with intracerebral hemorrhage in men. Haematologica. 2007;92(4):513-8.

16. Noris P, Perrotta S, Seri M, Pecci A, Gnan C Loffredo G, et al. Mutations in ANKRD26 are responsible for a frequent form of inherited thrombocytopenia: analysis of 78 patients from 21 families. Blood. 2011;117 (24):6673-80

17. Hou M, Andersson PO, Stockelberg D, Mellqvist UH, Ridell B, Wadenvik $\mathrm{H}$. Plasma thrombopoietin levels in thrombocytopenic states: implication for a regulatory role of bone marrow megakaryocytes. $\mathrm{Br}$ J Haematol. 1998;101(3):420-424

18. Williams WJ, Beutler E, Erslev AJ, Lichtman MA. Hematology. Fourth Edition. New York. McGraw-Hill Publishing Company, 1990.

19. Liang HP, Morel-Kopp MC, Curtin J, Wilson M, Hewson J, Chen W, Ward CM. Heterozygous loss of platelet glycoprotein (GP) Ib-V-IX variably affects platelet function in velocardiofacial syndrome (VCFS) patients. Thromb Haemost. 2007;98(6): 1298-308.

20. Kunishima S, Naoe T, Kamiya T, Saito H Novel heterozygous missense mutation in the platelet glycoprotein Ib beta gene associated with isolated giant platelet disorder. Am J Hematol. 2001; 68(4):249-55 